\title{
Electrochemical Instability of Metal-Organic Frameworks: In Situ Spectroelectrochemical Investigation of the Real Active Sites
}

Weiran Zhengs, Mengjie LiL», Lawrence Yoon Suk Lee*

Department of Applied Biology and Chemical Technology and the State Key Laboratory of Chemical Biology and Drug Discovery, The Hong Kong Polytechnic University, Hung Hom, Kowloon, Hong Kong SAR, China.

Corresponding Author: lawrence.ys.lee@polyu.edu.hk (L. Y. S. Lee) 


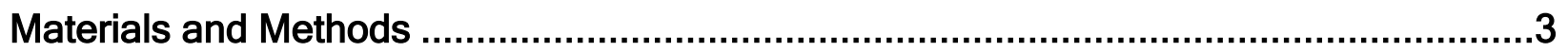

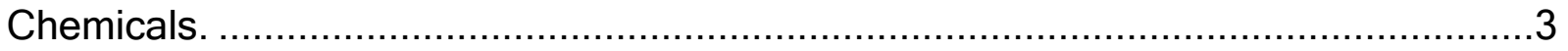

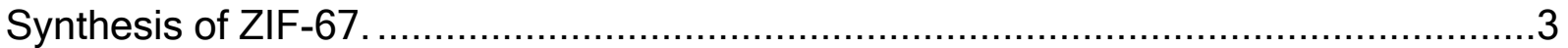

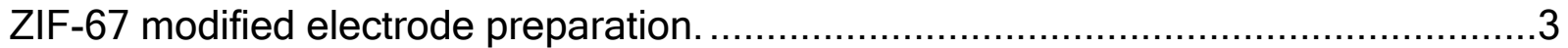

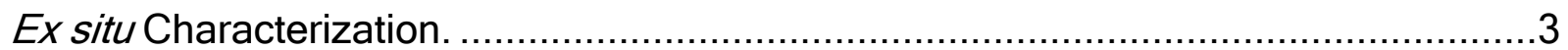

Electrochemical treatment and in situ spectroelectrochemistry studies.....................4

Calculation of OER turnover frequency (TOF) ................................................ 4

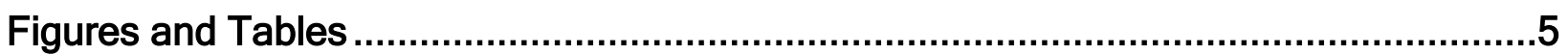

Figure S1 Nyquist plots of ZIF-67@CFP electrode after treated with CV by different

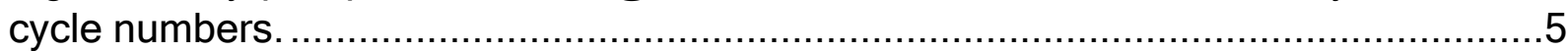

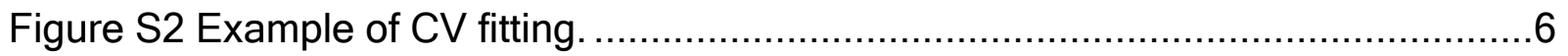

Figure S3 Kinetic aspect of OER using CV treated samples. ..................................6

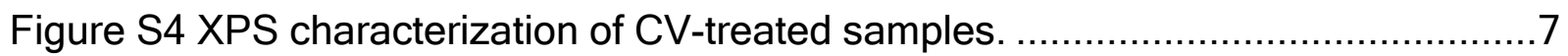

Table S1. Atomic ratios of surface species determined by the XPS survey spectra and

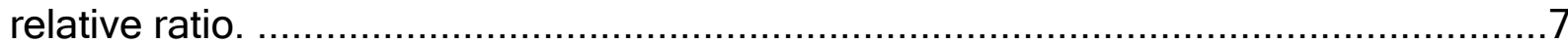

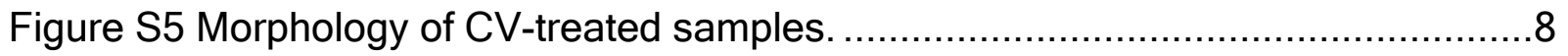

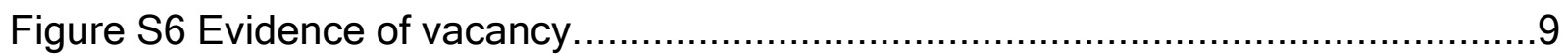

Figure S7 Device, integrated CV curves, and the reverse scan of in situ UV-vis and Raman spectroelectrochemistry (SPECE) ..................................................10

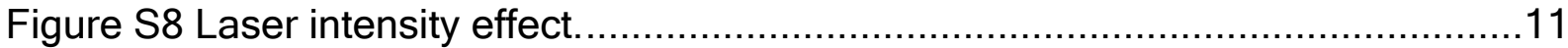

Figure S9 Kinetic aspect of OER using amperometric treated samples. ...................11

Figure S10 Morphology of amperometric treated samples. ...................................12 


\section{Materials and Methods}

Chemicals. Cobalt(II) nitrate hexahydrate $\left(\mathrm{Co}\left(\mathrm{NO}_{3}\right)_{2} \quad 6 \mathrm{H}_{2} \mathrm{O}, \quad 99.999 \%\right)$, hexadecyltrimethylammonium bromide (CTAB, >99.0\%), potassium hydroxide $(\mathrm{KOH}, \geq 99.97 \%)$, ethanol $(\mathrm{EtOH}, \geq 99.8 \%)$, Nafion ${ }^{\circledR} 117$ solution, and 2-methylimidazole (mIM, 99\%) were purchased from Sigma-Aldrich and used as received. The aqueous solution was prepared using double-deionized water (DI water, $\mathrm{R}>18.2 \mathrm{M} \Omega \mathrm{cm}^{-1}$ ) produced by MilliQ Water System (Millipore, USA). Carbon fiber paper electrode (CFP, HCP030 N, Chuxi, Shanghai, $3 \mathrm{~m} \Omega \mathrm{cm}^{-2}$ ) and graphite paper electrode (Sigri Great Lakes Carbon $\mathrm{GmbH}$ ) were washed using DI water and EtOH before use.

Synthesis of ZIF-67. ZIF-67 was prepared using a surfactant-assisted method. Typically, $174 \mathrm{mg}$ of $\mathrm{Co}\left(\mathrm{NO}_{3}\right)_{2} 6 \mathrm{H}_{2} \mathrm{O}$ was dissolved in $6 \mathrm{~mL}$ DI water with $9 \mathrm{mg} \mathrm{CTAB}$, and the mixture was rapidly injected into a $30 \mathrm{~mL}$ aqueous solution containing $1.944 \mathrm{~g} \mathrm{2-methylimidazole} \mathrm{(mIM).} \mathrm{The} \mathrm{final}$ mixture was vigorously stirred for $30 \mathrm{~min}$. at room temperature. After centrifugation, the collected solid was washed six times with ethanol to remove excessive CTAB and dried at room temperature to yield ZIF-67 as purple powder.

ZIF-67 modified electrode preparation. The catalyst ink for electrode modification was prepared by dispersing $35 \mathrm{mg}$ ZIF-67 powder in $1 \mathrm{~mL}$ EtOH. For CV, electrochemical impedance spectroscopic (EIS), and amperometric studies, the ink (50 $\mathrm{LL}, 1.75 \mathrm{mg}$ ZIF-67) was drop-cast on the surface of carbon fiber paper (CFP) electrode to prepare ZIF-67@CFP electrode. For in situ UV-Vis spectroelectrochemistry, the ZIF-67 was coated in the inner pores of the gold honeycomb electrode. For in situ Raman spectroelectrochemistry, the ink was mixed with 0.25 wt\% Nafion solution and coated on the graphite paper electrode.

Ex situ Characterization. The morphology of material was characterized by a field emission (FE) scanning electron microscope (SEM, TESCAN MAIA3) and a transmission electron microscope (TEM, JEOL Model JEM-2100F, 200 kV). The elemental composition analysis and distribution mapping were obtained using an energy dispersive spectrometer (EDS) equipped with the TEM. X-ray powder diffraction (XRD) patterns were collected on a SmartLab X-ray diffractometer by Rigaku (voltage $45 \mathrm{kV}$, current $200 \mathrm{~mA}$ ) and the surface species information was provided by Xray photoelectron spectroscopy (XPS) spectra, acquired using a Thermo Fisher ESCALAB 250Xi. The $\mathrm{C}$ 1s peak at $284.6 \mathrm{eV}$ was used for spectrometer calibration. The data were analyzed using CasaXPS software, with a background type of Spline Shirley and peak profile of GaussianLorentzian GL(30). The surface area analysis was done on a Micromeritics ASAP 2020 Plus Physisorption analyzer using $\mathrm{N}_{2}$. For each sample, we added $5 \mathrm{mg}$ of ZIF-67 to the 
ITO electrode and collected the material after $1 \mathrm{~h}$ amperometric test. The process was repeated 4 times to collect the treated ZIF-67 samples.

Electrochemical treatment and in situ spectroelectrochemistry studies. All electrochemical tests were carried using a three-electrode configuration in $1.0 \mathrm{M} \mathrm{KOH}$ electrolyte with $\mathrm{Pt}$ foil as the counter electrode and $\mathrm{Hg} / \mathrm{HgO}(1.0 \mathrm{M} \mathrm{KOH})$ as the reference electrode using a PARSTAT MC (PMC 1000/DC, Princeton Applied Research) potentiostat. The cyclic voltammetry was carried out at a scan rate of $10 \mathrm{mV} \mathrm{s}^{-1}$ and the EIS study was conducted from $100 \mathrm{kHz}$ to $10 \mathrm{~Hz}$ with an amplitude of $10 \mathrm{mV}$ at given potential values. For the potential conversion from $\mathrm{Hg} / \mathrm{HgO}(1.0 \mathrm{M}$ $\mathrm{KOH})$ reference electrode to the reversible hydrogen electrode (RHE), calibration experiment provided: $\mathrm{E}(v s . \mathrm{RHE})=\mathrm{E}(v s . \mathrm{Hg} / \mathrm{HgO}(1.0 \mathrm{M} \mathrm{KOH}))+0.9254 \mathrm{~V}$.

For the in situ UV-Vis spectroelectrochemistry study, an integrated spectroelectrochemical system (Pine Research Instrumentation, Inc., USA) was engaged using a gold honeycomb electrode as both the working and counter electrode and $\mathrm{a} \mathrm{Hg} / \mathrm{HgO}(1.0 \mathrm{M} \mathrm{KOH})$ as the reference electrode in $1.0 \mathrm{M} \mathrm{KOH}$ electrolyte. The ZIF-67 ink is coated onto the inner wall of the gold honeycomb tunnels $(0.1 \mu \mathrm{g}$ total). A staircase cyclic voltammetry (SCV) was applied with a potential amplitude of $5 \mathrm{mV}$ and a period of $20 \mathrm{~s}$.

A Raman spectrometer (Confocal Renishaw Micro-Raman Spectroscopy System) with a $785 \mathrm{~nm}$ stream-line laser excitation was used for the in situ Raman spectroelectrochemical study. Each spectrum was accumulated twice with $20 \mathrm{~s}$ exposure time and $0.5 \%$ of laser intensity (the power was reduced to $1.5 \mathrm{~mW})$. The $\mathrm{Hg} / \mathrm{HgO}(1.0 \mathrm{M} \mathrm{KOH})$ and Pt wire were employed as the reference and counter electrode, respectively. The ZIF-67@CFP electrode is used directly for in situ Raman spectroelectrochemical study. A CV method was engaged at a scan rate of $0.25 \mathrm{mV} \mathrm{s}^{-1}$.

Calculation of OER turnover frequency (TOF). The TOF values were calculated from the equation:

$$
\mathrm{TOF}=\frac{i}{4 F m}=\frac{i}{4 Q}
$$

where $i\left(\mathrm{~A} \mathrm{~s}^{-1}\right)$ is the total current at a given overpotential for OER, $F$ is the Faraday constant $\left(96,485 \mathrm{C} \mathrm{mol}^{-1}\right), 4$ is the transferred electron numbers to produce $1 \mathrm{O}_{2}, m(\mathrm{~mol})$ is the moles of electron accessible to Co sites, which can be estimated by the anodic charge $(Q)$ of $\mathrm{Co}^{2+}$ to $\mathrm{Co}^{3+}$ electrooxidation. 
Figures and Tables
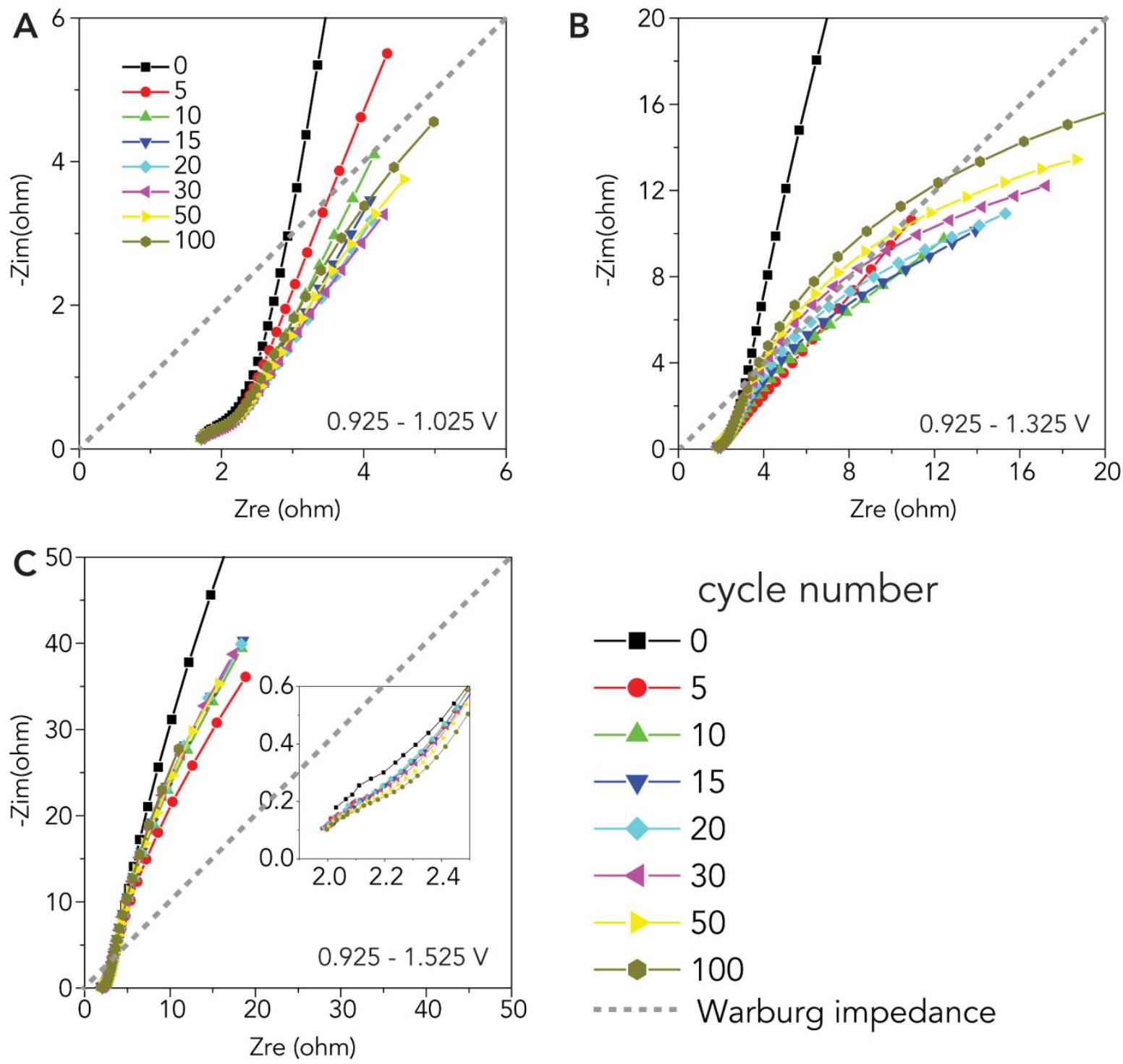

Figure S1 Nyquist plots of ZIF-67@CFP electrode after treated with CV by different cycle numbers. (A) $0.925-1.025 \vee(0.1)$, (B) $0.925-1.325 \vee(0.4)$, and (C) $0.925-1.525 \vee(0.6)$. The EIS experiments are carried out with an applied potential of $0.925 \mathrm{~V}$ vs. RHE in $1.0 \mathrm{M} \mathrm{KOH}$. 

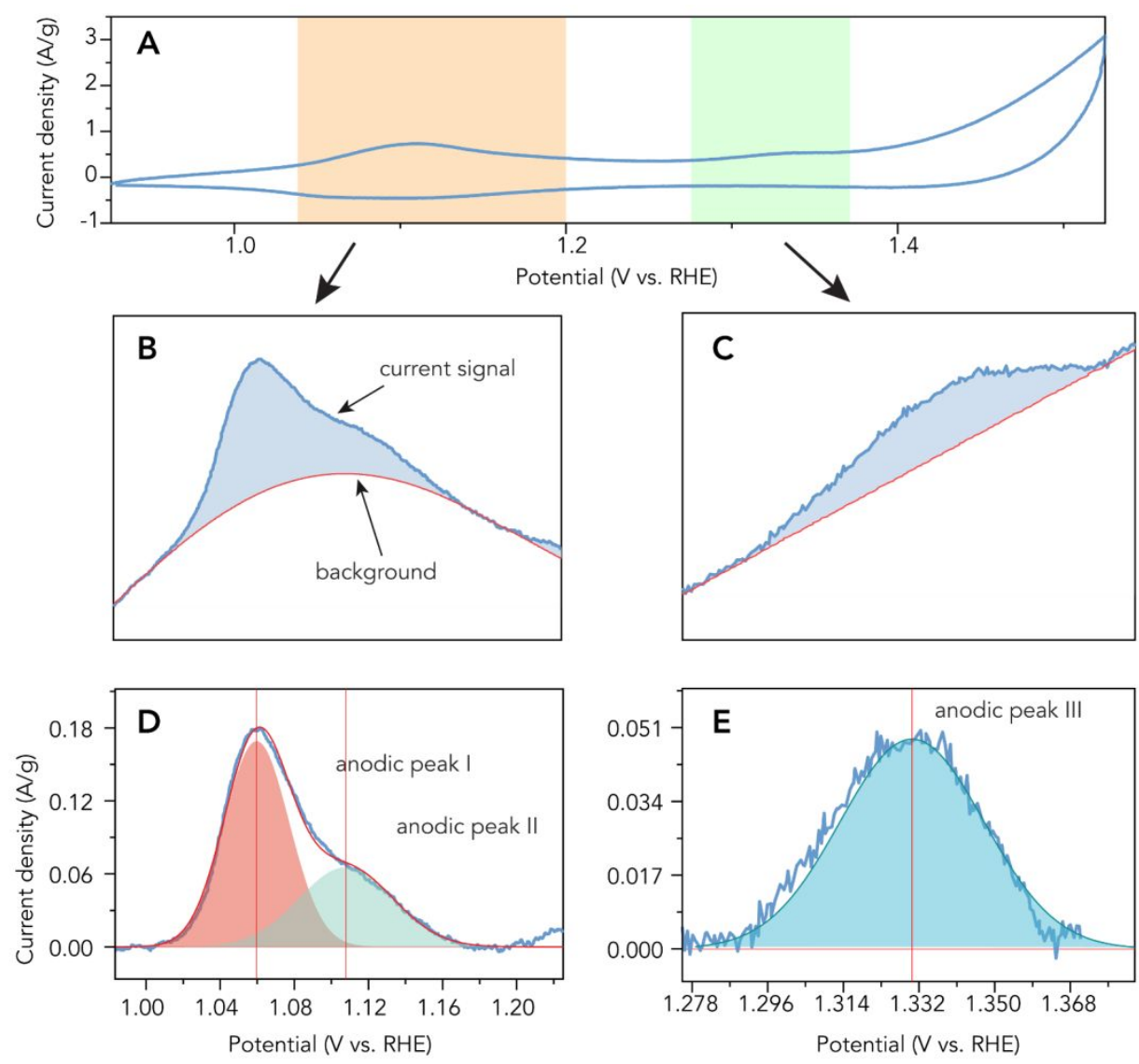

Figure S2 Example of CV fitting. (A) CV plot of the 10th cycle using the ZIF 67 modified electrode. $(B, C)$ Anodic region of $C V$ plot (0.925-1.525 V) with background baseline. (D, E) Peak-fitting results of background-substrate anodic peaks showing $\mathrm{D}$, anodic peak I and II and $\mathrm{E}$, anodic peak III.

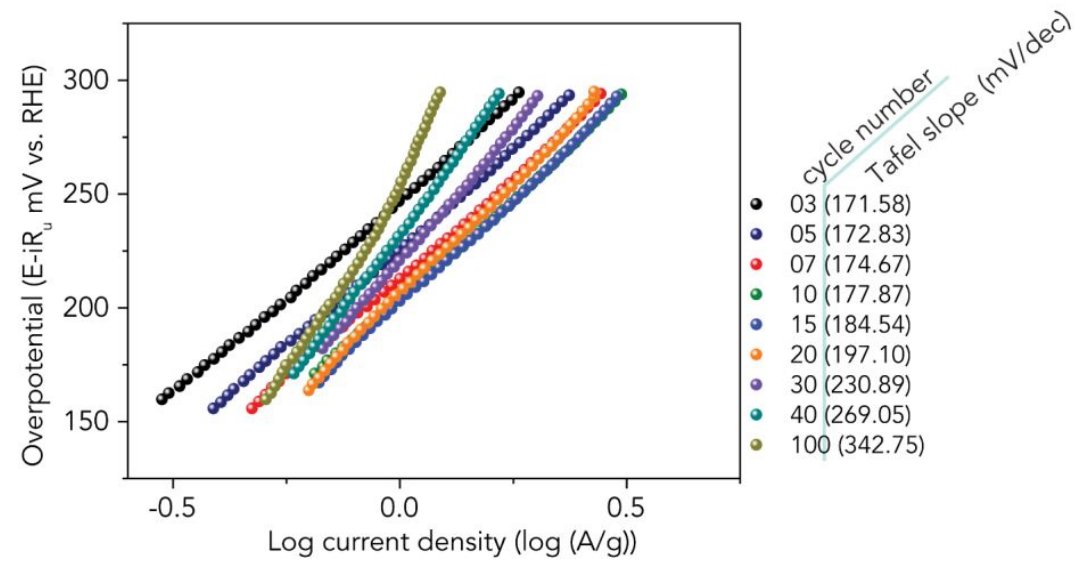

Figure S3 Kinetic aspect of OER using CV treated samples. Tafel plots of OER region after CV treatment between $0.925-1.525 \vee(0.6)$ for different cycle numbers $(3,5,7,10,15,20,30,40$, and 100). 

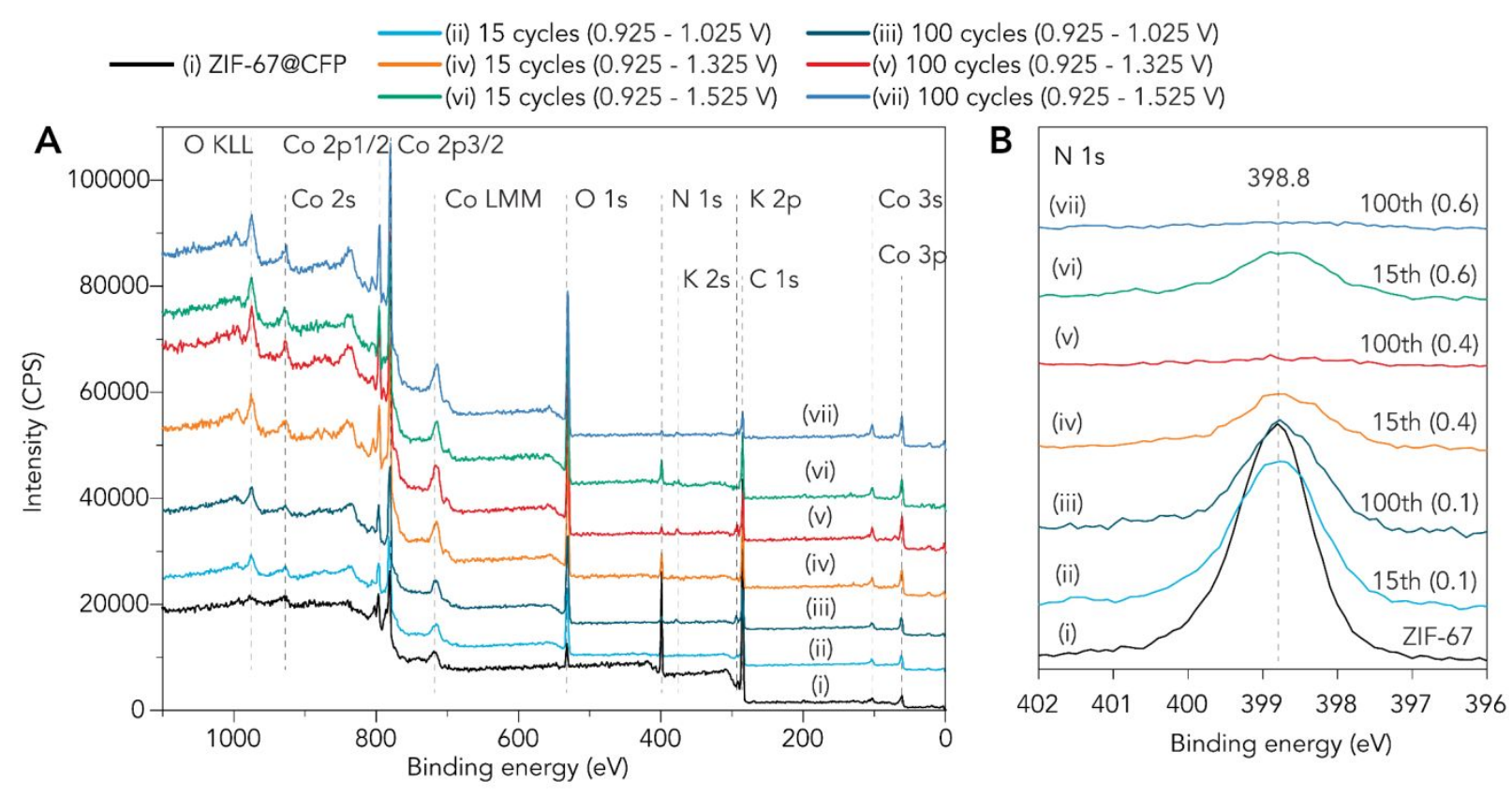

Figure S4 XPS characterization of CV-treated samples. (A) XPS survey spectra and (B) N 1s XPS spectra of pristine and CV-treated ZIF-67 with different cycles (15 and 100) between different potential window: $0.1(0.925-1.025 \mathrm{~V}), 0.4(0.925-1.325 \mathrm{~V})$, and $0.6(0.925-1.525 \mathrm{~V})$.

Table S1. Atomic ratios of surface species determined by the XPS survey spectra and relative ratio.

\begin{tabular}{l|l|l|l|l|l}
\hline Sample & $\mathrm{N}($ at. $\%)$ & $\mathrm{O}($ at. $\%)$ & $\mathrm{Co}$ (at. $\%)$ & $\mathrm{N} / \mathrm{Co}$ & $\mathrm{N} / \mathrm{O}$ \\
\hline Pristine ZIF-67@CFP & 18.95 & 3.94 & 5.22 & 3.63 & 4.81 \\
\hline $0.925-1.025 \mathrm{~V}(\mathbf{0 . 1}) 15$ cycles & 7.20 & 25.28 & 9.33 & 0.77 & 0.28 \\
\hline $0.925-1.025 \mathrm{~V}(\mathbf{0 . 1}) 100$ cycles & 4.41 & 32.79 & 11.44 & 0.39 & 0.13 \\
\hline $0.925-1.325 \mathrm{~V}(\mathbf{0 . 4}) 15$ cycles & 8.99 & 31.55 & 14.43 & 0.62 & 0.28 \\
\hline $0.925-1.325 \mathrm{~V}(\mathbf{0 . 4}) 100$ cycles & 3.65 & 46.74 & 17.49 & 0.21 & 0.078 \\
\hline $0.925-1.525 \mathrm{~V}(\mathbf{0 . 6}) 15$ cycles & 8.78 & 40.61 & 12.68 & 0.69 & 0.22 \\
\hline $0.925-1.525 \mathrm{~V}(\mathbf{0 . 6}) 100$ cycles & 4.28 & 51.34 & 20.65 & 0.21 & 0.083 \\
\hline
\end{tabular}




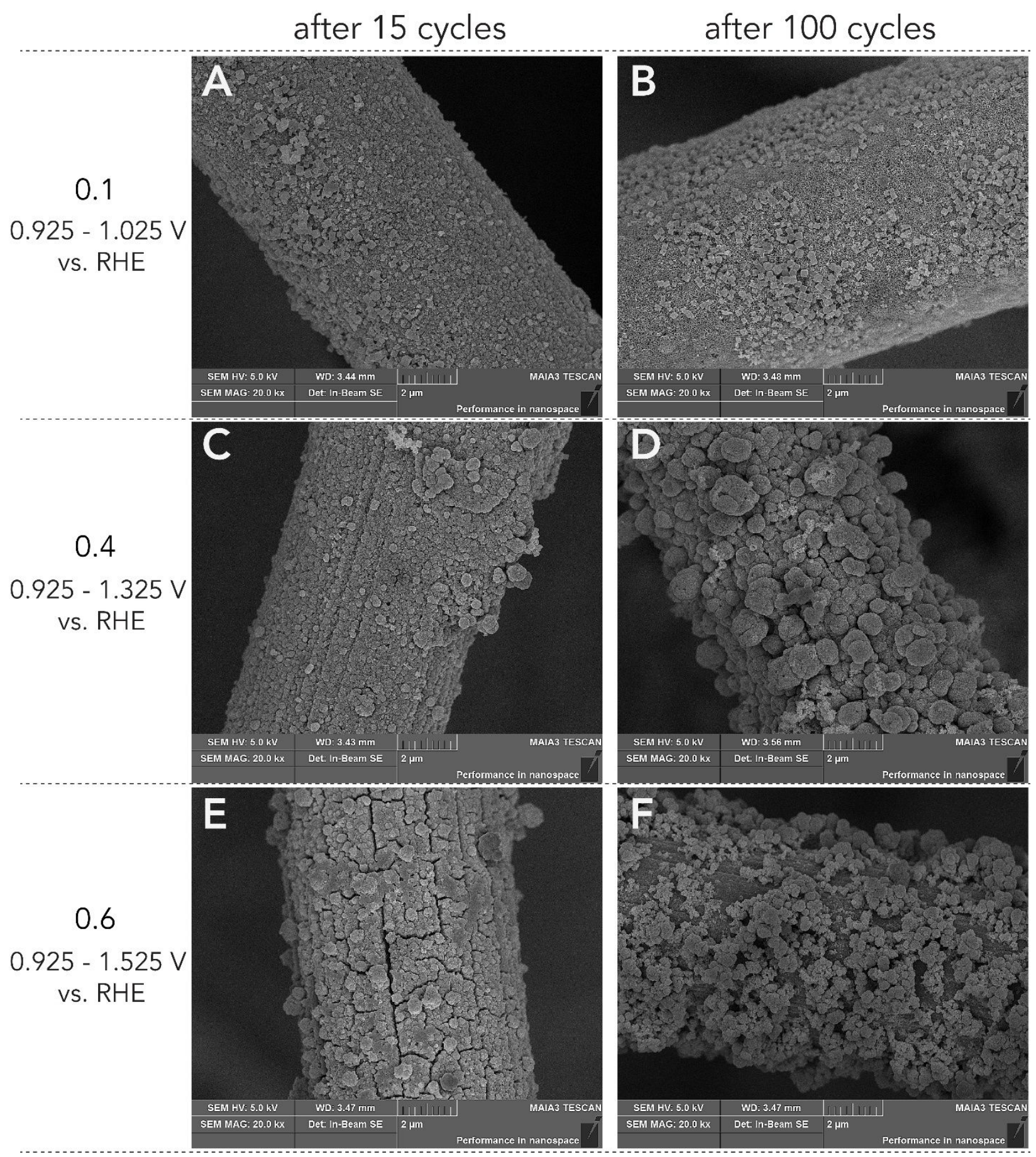

Figure S5 Morphology of CV-treated samples. SEM images of CV-treated ZIF-67@CFP electrode after different cycles (15 and 100) between different potential window: 0.1 (0.925 - 1.025 V), 0.4 $(0.925-1.325 \mathrm{~V})$ and $0.6(0.925-1.525 \mathrm{~V})$. 

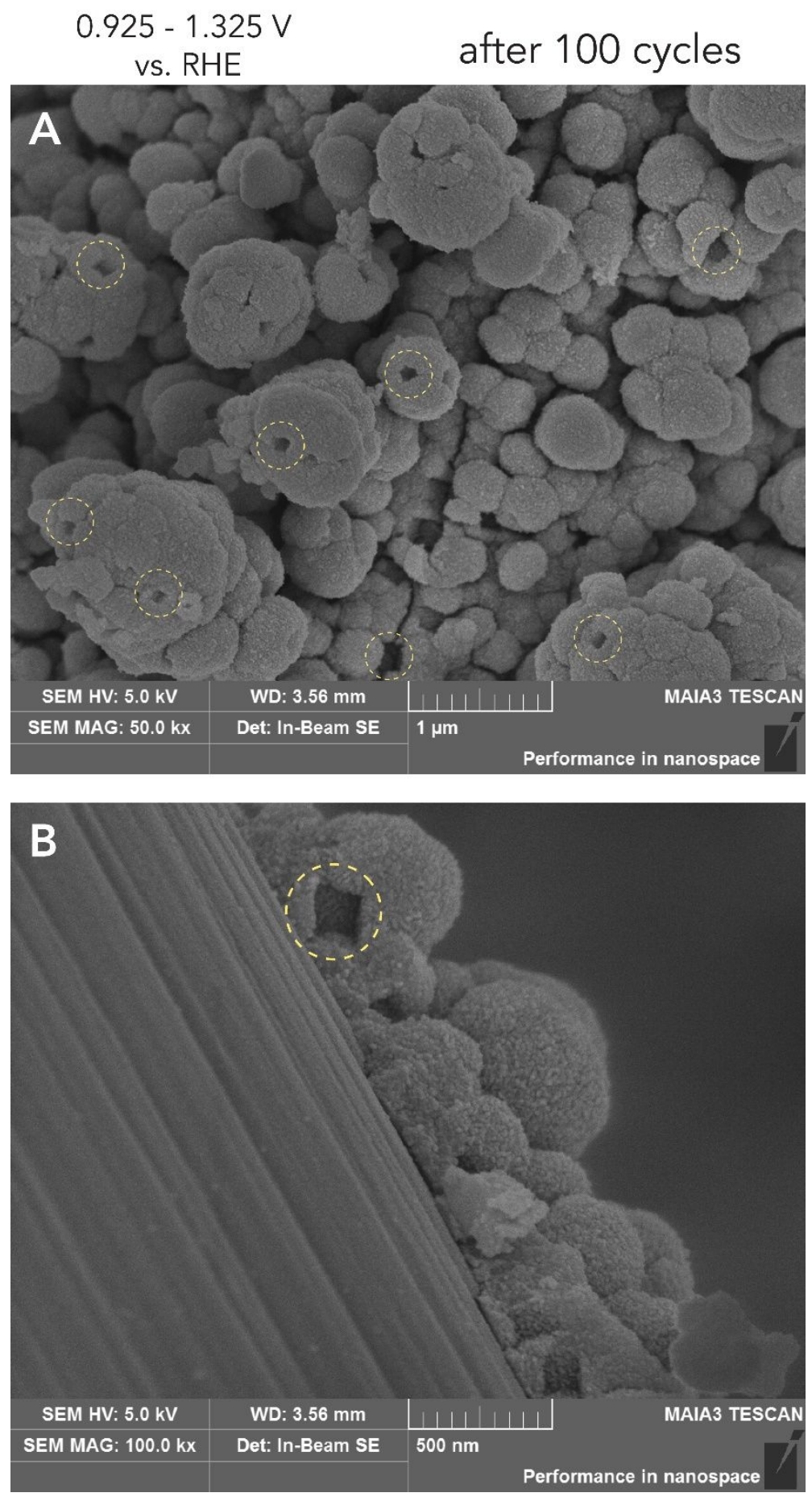

Figure S6 Evidence of vacancy. SEM images of CV-treated ZIF-67@CFP electrode between 0.925 and $1.325 \mathrm{~V}$ (0.4) for 100 cycles. (A) Top view. (B) Side view. The vacancies are highlighted in yellow cycles. 

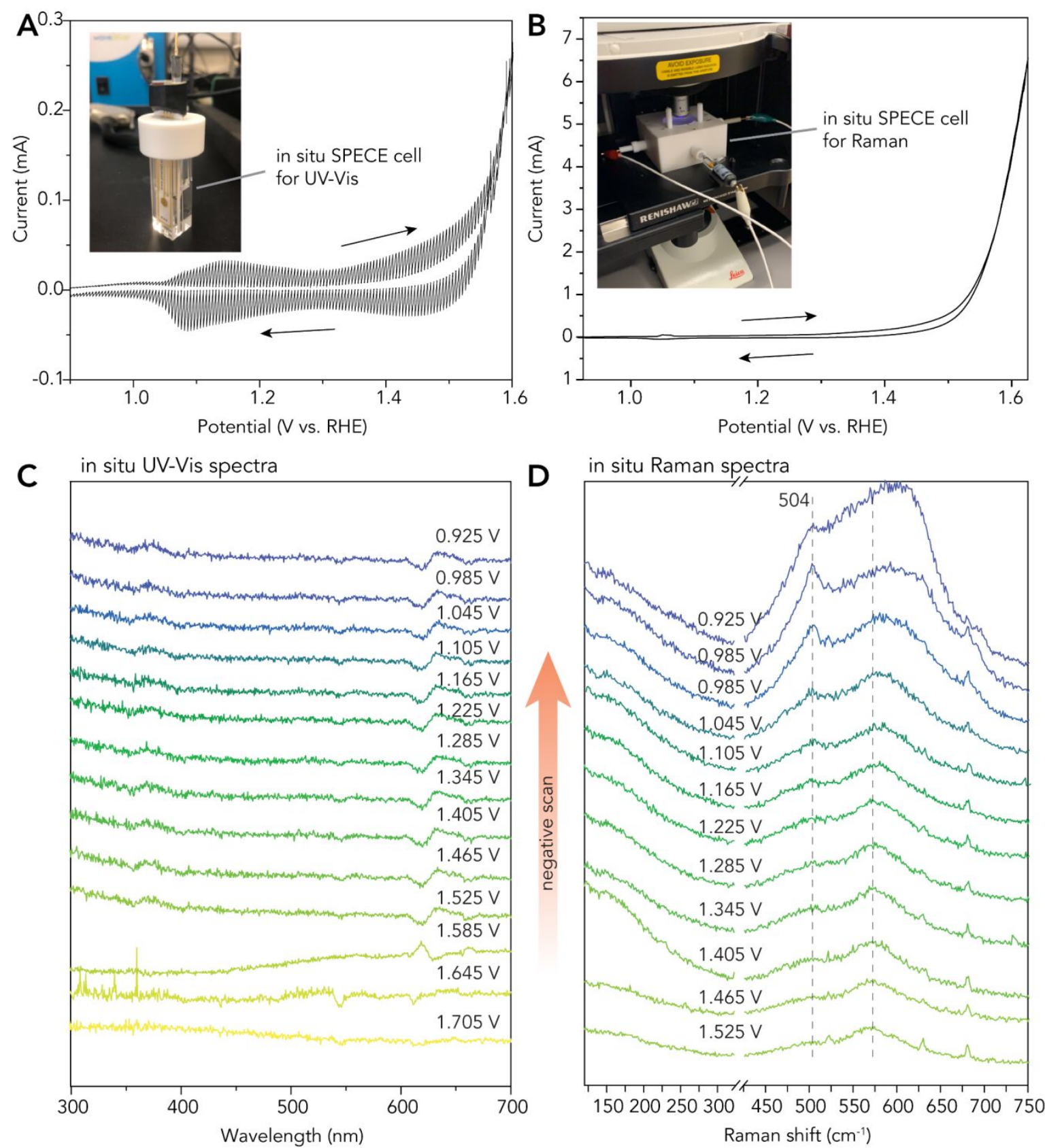

Figure S7 Device, integrated CV curves, and the reverse scan of in situ UV-vis and Raman spectroelectrochemistry (SPECE). (A) Integrated staircase CV plots of the in situ UV-Vis study, inset shows the in situ electrochemical cell for UV-Vis study. (B) Integrated CV plots of the in situ Raman study, inset shows the in situ electrochemical setup for Raman study. Reverse scan spectra of (C) UV-Vis and (D) Raman. 


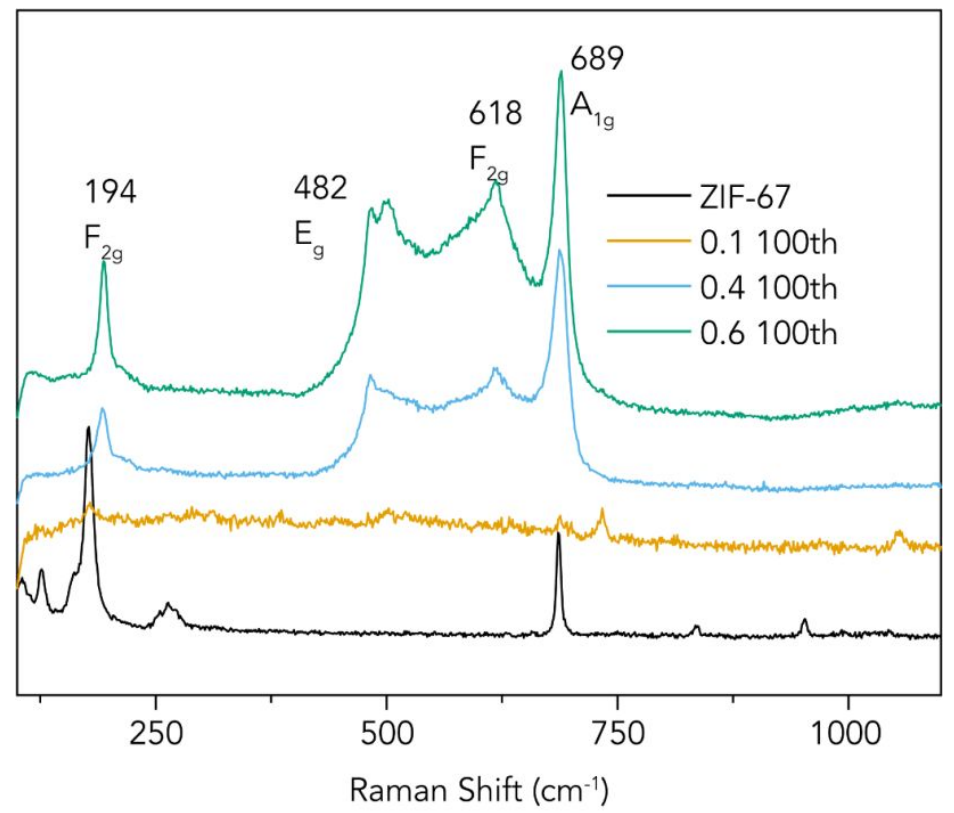

Figure S8 Laser intensity effect. Raman spectra of CV-treated ZIF-67 samples with an increased laser power (from $1.5 \mathrm{~mW}$ to $5.0 \mathrm{~mW}$ ). The peaks at 482 and $689 \mathrm{~cm}^{-1}$ match $\mathrm{Co}_{3} \mathrm{O}_{4} \mathrm{E}_{\mathrm{g}}$ and $\mathrm{A}_{1 \mathrm{~g}}$ vibration modes, confirming the phase transition from $\mathrm{Co}(\mathrm{OH})_{2} / \mathrm{CoOOH}$ to $\mathrm{Co}_{3} \mathrm{O}_{4}$ due to laser heating.

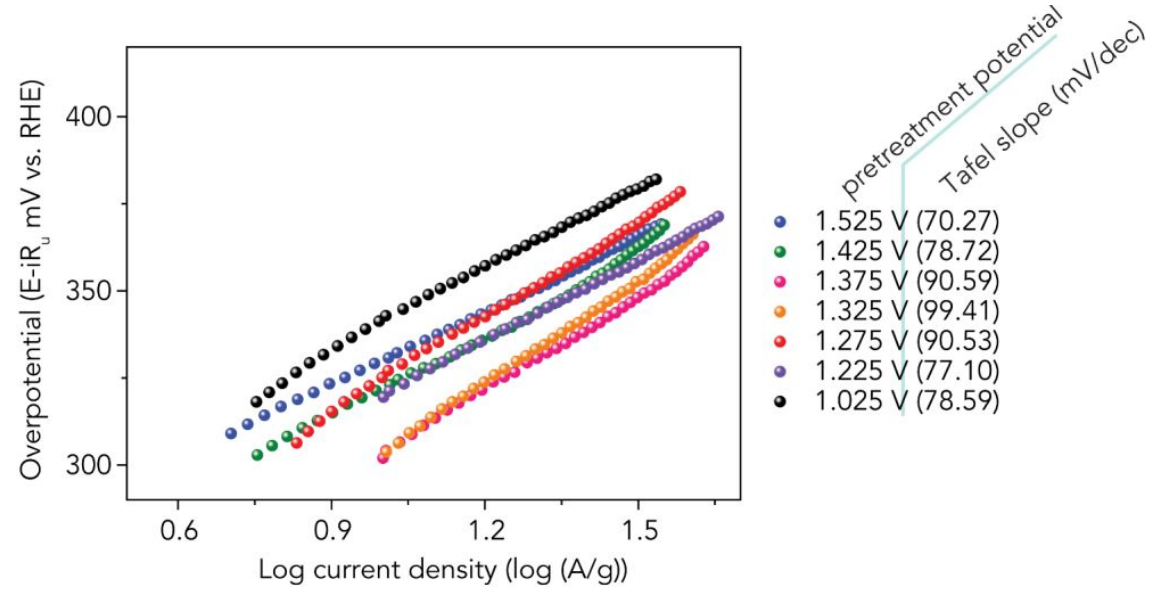

Figure S9 Kinetic aspect of OER using amperometric treated samples. Tafel plots of OER region after amperometric treatment at various potentials for $1 \mathrm{~h}$. 

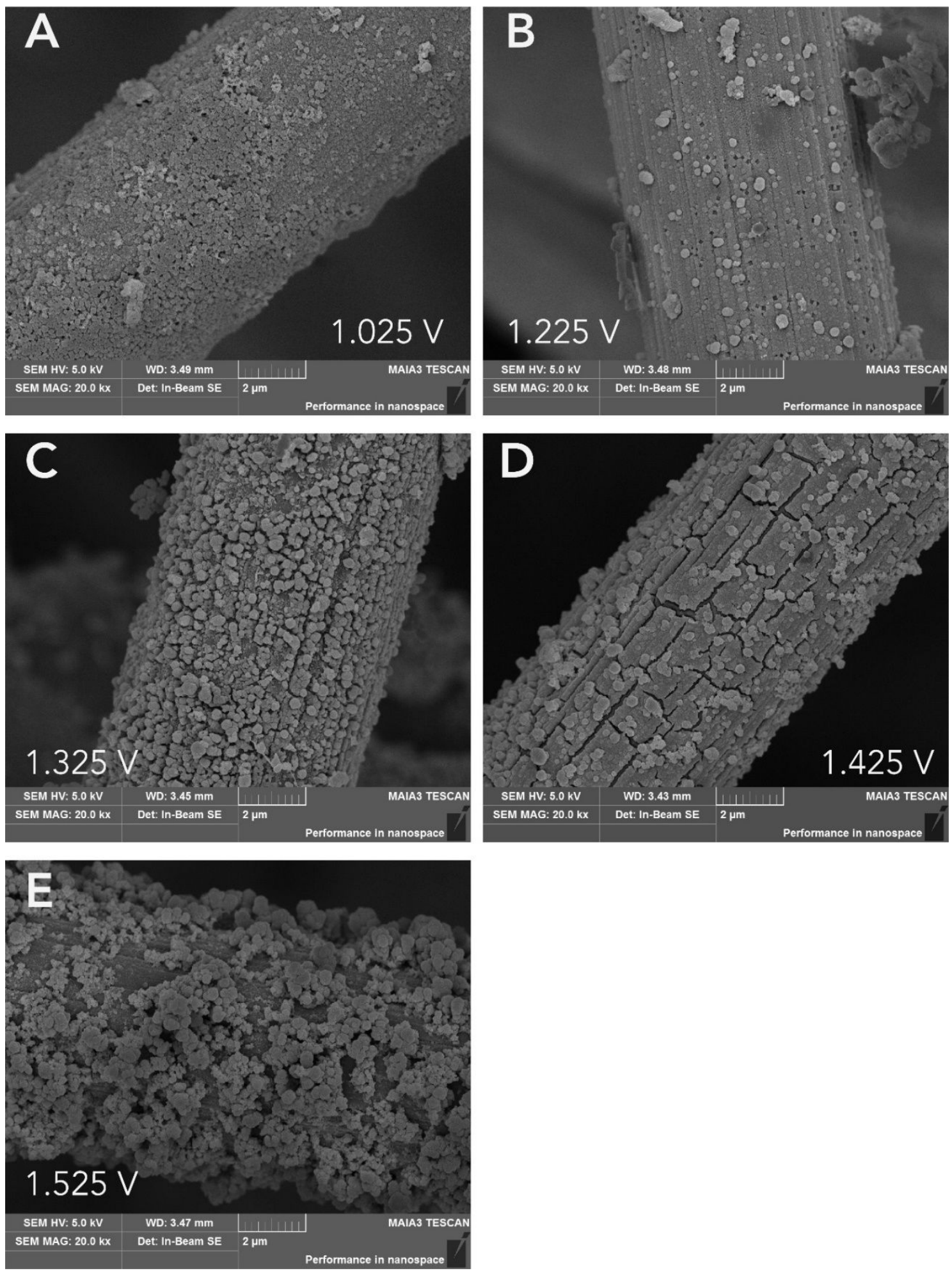

Figure S10 Morphology of amperometric treated samples. SEM images of ZIF-67@CFP electrode after amperometric treatment at various potential for $1 \mathrm{~h}$ : (A) $1.025 \mathrm{~V},(\mathrm{~B}) 1.225 \mathrm{~V}$, (C) $1.325 \mathrm{~V}$, (D) $1.425 \mathrm{~V}$, and $(\mathrm{E}) 1.525 \mathrm{~V}$. 